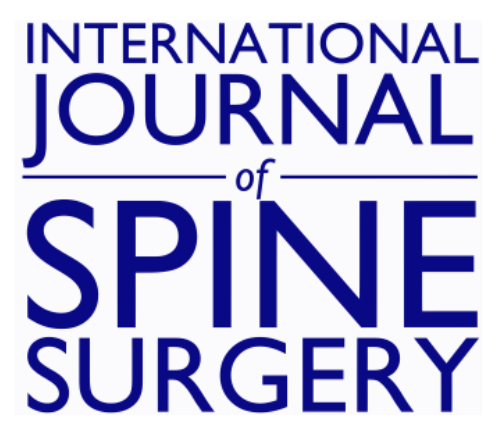

\title{
Clinical Outcomes Following Minimally Invasive Sacroiliac Joint Fusion With Decortication: The EVoluSIon Clinical Study
}

Donald Kucharzyk, Kyle Colle, Christopher Boone and Ali Araghi

Int J Spine Surg published online 25 February 2022

http://ijssurgery.com/content/early/2022/02/22/8185

This information is current as of April 26, 2023.

Email Alerts Receive free email-alerts when new articles cite this article. Sign up at:

http://ijssurgery.com/alerts

The International Journal of Spine Surgery

2397 Waterbury Circle, Suite 1,

Aurora, IL 60504, Phone: +1-630-375-1432 


\title{
Clinical Outcomes Following Minimally Invasive Sacroiliac Joint Fusion With Decortication: The EVoluSIon Clinical Study
}

\author{
DONALD KUCHARZYK, DO ${ }^{1}$; KYLE COLLE, DO ${ }^{2}$; CHRISTOPHER BOONE, MD ${ }^{3}$; AND ALI ARAGHI, DO ${ }^{4}$ \\ ${ }^{I}$ DK Orthopedics, Crown Point, IN, USA; ${ }^{2}$ Regional Brain and Spine, Cape Girardeau, MO, USA; ${ }^{3}$ Proliance Orthopaedics \& Sports Medicine, Bellevue, WA, USA; \\ ${ }^{4}$ The CORE Institute, Sun City West, AZ, USA
}

\begin{abstract}
Background: This report documents 1-year results of 250 patients enrolled in a prospective, multicenter study of a minimally invasive (MI) sacroiliac joint fusion (SIJF) system that uses decortication, graft placement, and joint fixation.

Methods: The cohort includes all patients enrolled in the EVoluSIon (EVSI) clinical study who had MI SIJF surgery and completed 1-year follow-up. Average age at baseline was 60.5 years, and 70.8\% were female. Sacroiliac (SI) joint-related pain duration was $\geq 2$ years in $56 \%$ of patients. Visual analog scale (VAS) SI joint pain, Oswestry Disability Index (ODI), quality of life, and opioid use were assessed preoperatively and at 1 year.

Results: At 1 year, the mean VAS pain demonstrated a significant reduction of more than 43 points, from 76.4 at baseline to $33.0(P<0.0001)$, with $72.2 \%$ of patients attaining the minimal clinically important difference (MCID, $\geq 20$-point improvement). Mean ODI scores also significantly improved from 54.4 at baseline to 30.5 at 1 year $(P<0.0001)$, with $62.5 \%$ of patients achieving the MCID ( $\geq 15$-point improvement). Prior to surgery, $62.7 \%(126 / 201)$ of patients were taking opioids or other narcotics, but by 1 year postsurgery, only $26.9 \%$ (54/201) of patients reported using these medications, representing a significant $57.1 \%$ decrease in narcotic/opioid use $(P<0.0001)$. Fusion of the SI joint was seen in $68.7 \%$ of patients. Few procedural complications were reported. In all, there were $8(8 / 250)$ serious procedure-related events, including 1 device malposition observed on the day of surgery that required replacing the superior screw with a shorter screw.

Conclusions: Analysis of patients treated with MI SIJF in the EVSI study demonstrated that the procedure can be performed safely and results in significant improvements in pain, quality of life, and opioid use at 1 year as well as causing fusion in the majority of patients.
\end{abstract}

Level of Evidence: 2.

Clinical Relevance: MI SIJF differs from most procedures currently being performed in that it applies true orthopedic principles with decorticating, bone grafting, fusion, and placement of implants perpendicular to the joint for greatest stability. The 12-month data show improvement in functionality, reduction in pain, and, most notably, a reduction in narcotic usage, which is important considering the ongoing opioid epidemic.

Minimally Invasive Surgery

Keywords: sacroiliac joint dysfunction, sacroiliac joint fusion, SI joint, SI joint arthrodesis, minimally invasive surgery, sacroiliac, fusion, arthrodesis

\section{INTRODUCTION}

Minimally invasive (MI) sacroiliac joint fusion (SIJF) has become a preferred treatment alternative for pain originating in the sacroiliac (SI) joint that is refractory to conservative treatment. ${ }^{1-4}$ With the increasing popularity of this procedure, there are now more than 20 implantable devices indicated for SI joint fixation, yet there is scant published clinical data to understand the merits or comparative effectiveness of the different systems that are available. Within clinical publications pertaining to MI SIJF, there are even less data regarding actual fusion rates. ${ }^{5,6}$
An MI SIJF-implantable device system incorporating decortication, bone grafting, and fixation with threaded implants has demonstrated encouraging results in smaller case series. ${ }^{4,7}$ In the present study, 250 patients were enrolled in a prospective, multicenter study to evaluate pain, quality of life, opioid use, and radiographic fusion outcomes over the course of 2 years with an MI SIJF system that incorporates a graft site preparation step using a proprietary decortication tool. This report presents results of a cumulative analysis for all patients enrolled in the study with data available at the interim 1-year timepoint and serves as an update to the 6-month report on the first 50 patients enrolled in this study. ${ }^{8}$ 


\section{METHODS}

\section{Study Design}

This interim report includes all patients participating in a prospective, multicenter evaluation of the commercially available SImmetry Sacroiliac Joint Fusion System (Surgalign Spine Technologies, Deerfield, IL) in the EVoluSIon (EVSI) study, registered on clinicaltrials.gov as NCT02074761. The EVSI study enrolled 250 patients at 23 centers in the United States. The study is now closed to enrollment and is in follow-up. The protocol was approved either by a central institutional review board (IRB) or by each participating center's IRB, and written informed consent was obtained from all patients prior to enrollment. Patients were screened from each investigator's population of patients indicated for MI SIJF. Patients receiving or seeking workers' compensation benefits were excluded. The key inclusion criteria for participation were 18 years or greater; at least 6 months of nonoperative management of SI joint pain; 3 positive provocative tests (such as FABER, gaenslen, compression, thigh thrust, and distraction) $;{ }^{9}$ at least 1 positive diagnostic SI joint injection that resulted in at least a $50 \%$ reduction in pain within 6 months prior to implantation; ${ }^{10,11}$ visual analog scale (VAS) SI joint pain score of 60 or greater; and Oswestry Disability Index (ODI) score of at least 40. Patients $(n=23)$ who did not meet minimum eligibility requirements for baseline VAS or ODI scores were not excluded from the analysis to limit influence of bias from analysis. These and any other protocol noncompliance events were reported to the investigating sites' IRB, as required.

\section{Surgical Procedure}

The MI SIJF surgical procedure included use of the SImmetry System in accordance with the approved labeling. The implant system includes a $12.5-\mathrm{mm}$ diameter cannulated implant placed through the location of decortication and an $8.5-\mathrm{mm}$ diameter antirotational implant for mechanical stability. Both devices are threaded implants made of Ti-6Al-4V titanium alloy with a microscale texture designed for osseointegration.

The procedure has been described in detail elsewhere. ${ }^{3,12,13}$ Briefly, 4 steps are followed: MI lateral access, joint preparation via decortication, bone graft placement, and implant delivery (Figure 1). Joint preparation is a key step in the MI SIJF procedure with the SImmetry System using a proprietary decorticator to remove cartilage and prepare up to $50 \%$ of the SI joint surface as an active bleeding fusion bed for bone graft material. Bone graft is then packed into the decorticated area, before the cannulated implant is placed across

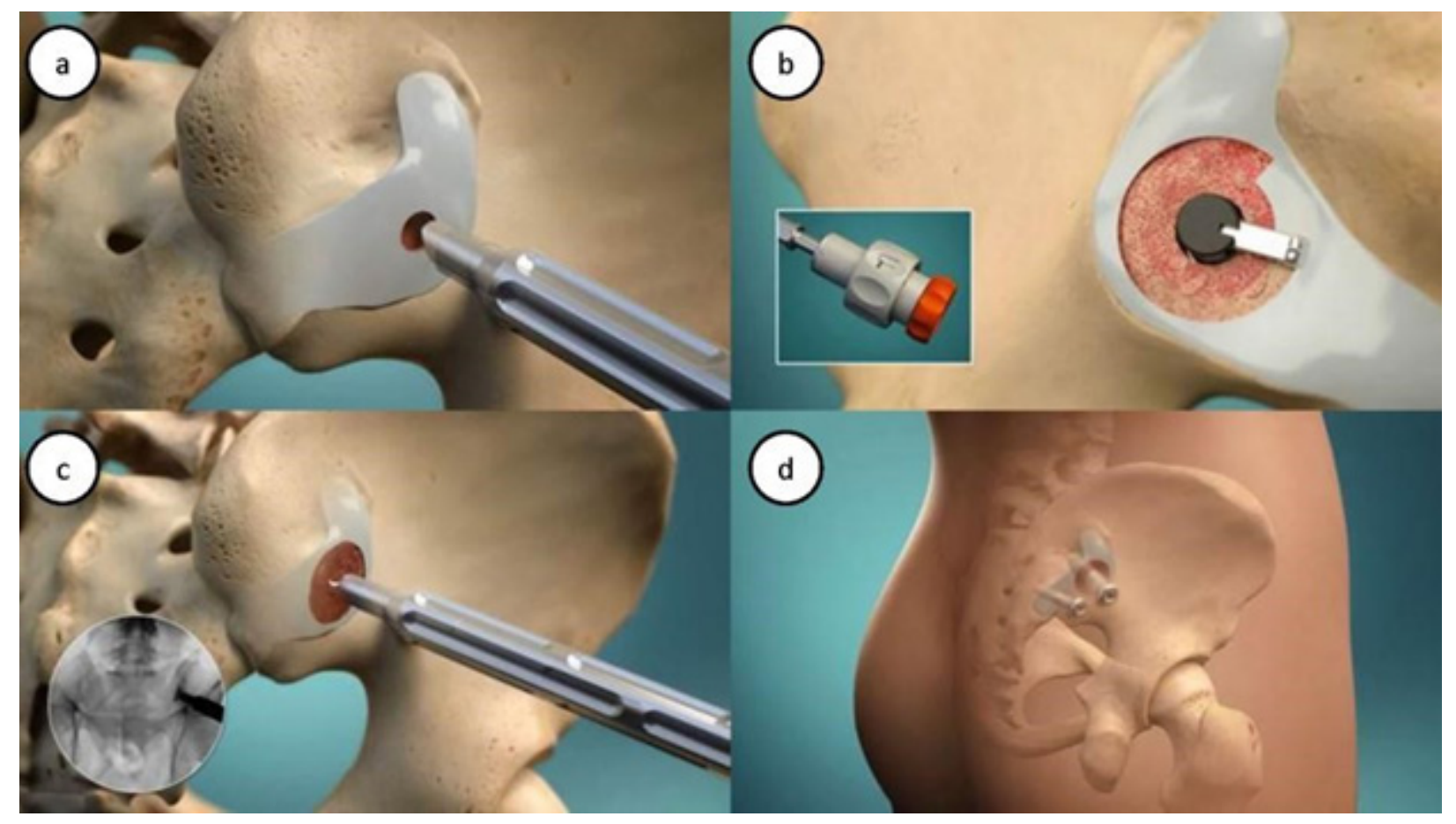

Figure 1. (A) Minimally invasive lateral access of the sacroiliac joint; (B) decortication of the joint (inset: user-interface of decorticator instrument); (C) delivery of bone graft into the actively bleeding fusion bed (inset: fluoroscopic image during decortication); and (D) final placement of the threaded implant through the area of decortication and a secondary device for stability. 
the area of decortication. A secondary antirotational implant is then placed for biomechanical stability. ${ }^{14}$

\section{Outcomes}

Baseline data collection included medical history, prior treatments and surgeries, provocative maneuvers to confirm the diagnosis of SI joint dysfunction, diagnostic SI joint injections within 6 months prior to implantation (sites followed their standard of care for diagnostic injections; however, at least 1 diagnostic injection was required to be within 6 months of the SImmetry procedure; this was needed to diagnose and treat SI joint dysfunction), and pain medication usage. Patients were asked to complete a questionnaire, which included self-evaluation of various elements: (1) SI joint pain as measured by a VAS from 0 to 100 , where 0 represented no pain and 100 represented the worst possible pain; (2) disability due to low back pain as measured by the validated ODI survey; and (3) quality of life through the EQ-5D questionnaire. The patient questionnaires and pain medication usage were collected preoperatively and at specified timepoints of 6 weeks, 3 months, 6 months, and 12 months postoperatively. Patient questionnaires were self-reported and completed by each patient prior to meeting with the investigator to limit bias. Patient qualification and endpoint data were monitored, and the source was verified for accuracy in reporting and compliance. Data were collected and reported via electronic database (Fortress Medical Systems, Hopkins, MN).

VAS pain and ODI reductions were also defined in terms of minimal clinically important difference (MCID) of 20 points on the VAS scale and 15 points on the ODI scale. ${ }^{3}$ Multiple secondary analyses were defined in the protocol, including comparison of baseline and procedural data to outcomes of pain reduction and fusion. EQ-5D is a widely used standardized measure of health status consisting of a patient-completed questionnaire with 5 quality-of-life questions that can be combined into a single index of "preference weights," representing the time trade-off of current health. A VAS-like pain scale is also included, where 0 is the worst imaginable health state and 100 is the best imaginable health state. $^{15,16}$

SIJF was assessed by an independent core laboratory at the 12-month follow-up visit via thin-slice $(<2$ $\mathrm{mm}$ ) computed tomography (CT), viewing 4 consecutive cuts on both coronal and sagittal reconstructions. Radiographic evidence of fusion was defined as presence of a continuous segment of bridging bone that extends from the sacrum to the ilium. This qualitative
Table 1. Classification of fusion based on radiographic evidence.

\begin{tabular}{lc}
\hline Fusion Category & \multicolumn{1}{c}{ Description } \\
\hline $\begin{array}{l}\text { Fusion } \\
\text { No fusion }\end{array}$ & $\begin{array}{l}\text { Presence of bridging across the treated joint } \\
\text { No bridging bone visible or images were reported as } \\
\text { "Indeterminate" or "Unable to Assess" }\end{array}$ \\
\hline
\end{tabular}

assessment of fusion status was performed by trained, independent radiologists, including 2 evaluators and an adjudicator (in case of a disagreement). Fusion status was classified according to the following preestablished criteria shown in Table 1. Examples of each fusion category using representative CT scans from patients evaluated in this study are shown in Figure 2.

In cases where an assessment could not be made from the available images due to technical factors (eg, obscured anatomy, poor contrast, or high parallax), the assessment was graded as "Indeterminate." If an assessment could not be made due to missing films, the assessment was graded as "Unable to Assess."

\section{Statistical Analyses}

Predefined statistical analyses described in the protocol include the primary endpoint analysis of VAS SI joint pain scores beginning with the 6-month follow-up visit. Pain relief postimplantation was evaluated at each follow-up interval with descriptive statistical analyses (mean, standard deviation, median, minimum and maximum).

Statistical analyses were performed using paired $t$ tests to assess improvements following treatment using the preoperative and follow-up VAS, ODI, and qualityof-life measures for each patient over time. Confidence

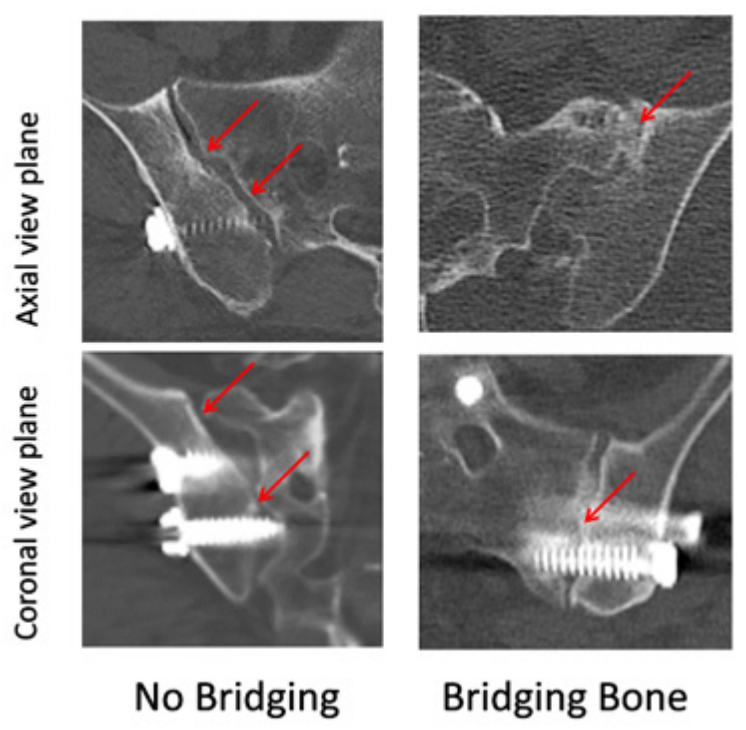

Figure 2.. Examples of bridging bone categories. 
intervals of $95 \%$ on the mean changes from the preoperative result in ODI and VAS scores were computed. Procedural data were summarized with descriptive statistics and 95\% confidence intervals. Subgroup analyses for the primary endpoints were planned to determine whether baseline or procedural characteristics differentiate subjects in terms of outcomes. Logistic and linear regression models were used for these analyses for binary vs continuous endpoints.

The McNemar test was used to determine the significance of the decreased usage of medications at the 1-year follow-up visit. This test compares the rate of patients who stop using opioids as a result of the SIJF procedure to the rate of patients who start taking opioids following the procedure; the relative rates are used to determine significance between groups. ${ }^{17}$ All statistical analyses were performed using the SAS statistical software package version 9.4 (Cary, NC).

\section{RESULTS}

\section{Patient Demographics and Baseline Characteristics}

The cohort for this interim analysis includes all patients enrolled in the study who had MI SI joint fusion surgery and completed a 1-year follow-up visit.
The 250 patients were enrolled at 23 institutions. The average age at baseline was 60.5 years; the majority were women (70.8\% [177/250]); and the duration of SI joint-related pain prior to surgery was at least 2 years in $56.0 \%$ of patients. Over half of the patients never used tobacco, and only $13.6 \%$ (34/250) were currently using cigarettes at the time of enrollment. Many patients had a prior lumbar surgery.

The mean SI joint VAS pain score at baseline was $76.6 \pm 11.9$ (mean $\pm \mathrm{SD} ; n=249$; range: $30-100)$, and the ODI was $54.5 \pm 13.1(n=249$; range 18-92). While inclusion criteria required a baseline VAS SI joint pain score of 60 or greater and a baseline ODI score of at least 40, 23 patients who did not meet minimum VAS and ODI scores were not excluded from data sets to limit influence from the analysis. Sixty-two percent of patients were taking opioids or other narcotics prior to surgery. Baseline characteristics are further summarized in Table 2.

\section{Perioperative Results}

Two hundred and fifty patients underwent planned SIJF surgery with the SImmetry System. Treatment information was collected on 250 procedures; $97.2 \%$ were unilateral with the remaining procedures

Table 2. Baseline characteristics of study participants.

\begin{tabular}{|c|c|c|}
\hline Demographics & $N$ & Summary \\
\hline Age at procedure, mean \pm SD (range) & 249 & $60.5 \pm 12.8(21-87)$ \\
\hline Female, $\%(n)$ & 250 & $70.8 \%(177)$ \\
\hline BMI $\left(\mathrm{kg} / \mathrm{m}^{2}\right)$, mean $\pm \mathrm{SD}($ range $)$ & 250 & $30.1 \pm 6.8(17-56)$ \\
\hline Duration of SI joint pain, $\%(n)$ & 250 & \\
\hline 6 months to 1 year & & $18.0 \%(45)$ \\
\hline $1-2$ years & & $26.0 \%(65)$ \\
\hline$<2$ years & & $56.0 \%(140)$ \\
\hline Taking narcotics/opioids, \% (n) & 250 & $62.0 \%(155)$ \\
\hline Prior SI joint treatment, \% $(n)$ & 250 & \\
\hline Acupuncture & & $8.8 \%(22)$ \\
\hline Biofeedback & & $0 \%$ \\
\hline Bracing & & $30.0 \%(75)$ \\
\hline Chiropractic care & & $27.6 \%(69)$ \\
\hline Physical therapy & & $88.0 \%(220)$ \\
\hline Prior SI joint Fusion & & $8.0 \%(20)$ \\
\hline SI joint steroid injection & & $93.2 \%(233)$ \\
\hline Sacral nerve ablation & & $10.0 \%(25)$ \\
\hline TENS unit & & $22.4 \%(56)$ \\
\hline Prior lumbar spine procedures, \% (n) & 250 & \\
\hline Lumbar total disc replacement & & $0 \%$ \\
\hline Lumbar decompression & & $13.2 \%(33)$ \\
\hline Lumbar discectomy & & $11.2 \%(28)$ \\
\hline Lumbar laminectomy & & $17.2 \%(43)$ \\
\hline Lumbar fusion & & $36.8 \%(92)$ \\
\hline VAS pain score, mean \pm SD (range) & 249 & $76.6 \pm 11.9(30-100)$ \\
\hline Oswestry Disability Index, mean \pm SD (range) & 249 & $54.5 \pm 13.1(18-92)$ \\
\hline $\mathrm{EQ}-5 \mathrm{D}$, mean $\pm \mathrm{SD}($ range $)$ & 246 & \\
\hline EQ-5D preference weights (formerly "time trade-off") & & $0.52 \pm 0.17(-0.11-0.83)$ \\
\hline EQ-5D VAS (scale of 0 to 100 ) & & $60.22 \pm 23.03(0.00-100.00)$ \\
\hline
\end{tabular}

Abbreviations: SI, sacroiliac; VAS, visual analog scale. 
Table 3. Perioperative data.

\begin{tabular}{lccc}
\hline Procedure Measure & $\boldsymbol{N}$ & Mean \pm SD & Range \\
\hline Procedure duration, min & 248 & $54.2 \pm 24.8$ & $15-158$ \\
Total contrast used, cc & 235 & $2.0 \pm 8.0$ & $0-100$ \\
Estimated blood loss, cc & 249 & $53.9 \pm 107.5$ & $0-1500$ \\
Length of hospital stay, d & 248 & $0.8 \pm 0.7$ & $0-4$
\end{tabular}

bilateral. The mean procedure time was 54 min (range 15-158 min), and the mean estimated blood loss was $54 \mathrm{cc}$. The mean length of hospital stay was 0.8 days, with $92 \%$ of patients discharged within 1 day of procedure. Perioperative data are summarized in Table 3.

\section{Outcomes}

Two hundred and fifty patients were enrolled in the study at 23 sites. Of these, 80.4\% (201/250) were available at the 1-year visit, though not every metric was available for all these patients. Reasons for incomplete follow-up include the following: missed visits $(n=28)$, withdrawal of consent $(n=8)$, lost to follow-up $(n=10)$, physician request for withdrawal $(n=1)$, death unrelated to SI joint $(n=1)$, and device explant $(n=1)$. In addition, the number of completed subject questionnaires may differ from completed follow-up visits due to patient compliance when completing the questionnaires.

Investigating 1-year follow-up via paired analysis, mean VAS SI joint pain had decreased by nearly 44 points, from 76.4 at baseline to 33.0 at the 1-year follow-up $(n=194)$. This represents a statistically significant $56.8 \%(P<0.0001)$ reduction in pain, with $72.2 \%(140 / 194)$ of patients attaining the MCID ( $\geq 20$-point improvement).

The ODI results also demonstrated a significant improvement in disability scores after SI joint fusion surgery. Mean ODI scores improved from 54.4 at baseline to 30.5 at 1 year $(n=192)$, a $43.9 \%$ change for responding patients $(P<0.0001)$, with $62.5 \%$ (120/192) of patients achieving the MCID ( $\geq 15$-point improvement).

In addition to improvements in pain and disability, a significant decline in opioid and narcotic use was reported. Using a paired analysis approach, prior to surgery, $62.7 \%(126 / 201)$ of patients were taking opioids or other narcotics. By 1-year postsurgery, the number of patients taking opioids or narcotics had decreased to $26.9 \%$ (54 of 201 patients reporting; $P<$ 0.0001 ) representing a $57.1 \%$ reduction in the number of patients using narcotics/opioids.

A decrease in nonopioid pain medication use was also reported. At baseline, $51.7 \%$ of patients were taking nonsteroidal anti-inflammatory drugs (104/201), 37.3\% were taking other nonopioid analgesics $(75 / 201)$, and $9.0 \%$ were taking steroids for pain control (19/201). The percentage of patients using NSAIDS, analgesics, and steroids at the 1-year follow-up had decreased to $14.4 \%, 9.0 \%$, and $0 \%$, respectively.

A quality-of-life assessment using the EQ-5D questionnaire was collected at baseline and at the 1-year follow-up visit. The EQ-5D questionnaire included a VAS component. Patients were asked to rank their state of health using a VAS scale of 0 to 100 , with 100 being the best possible health. In paired analysis, the average score at baseline was 60.9. By the time of their 1-year visit, patients ranked their state of health at $72.8(n=$ 190). This 11.9 index change from baseline is a statistically significant $(P<0.0001)$ improvement.

At 1 year, $68.7 \%$ of patients showed fusion of the SI joint and $31.3 \%$ of patients showed no fusion (ie, did not show evidence of fusion or images were reported as "Indeterminate" or "Unable to Assess").

\section{Complications}

Few procedural or device-related serious adverse events (SAEs) were reported by the time of the 1-year follow-up. In all, the occurrence of serious procedure-related events was $3.2 \%$ (8/250), including 1 device malposition observed on the day of surgery that required replacement of the superior screw with a shorter screw (also noted as a device-related event). The other procedure-related SAEs reported within this time frame included back pain, a hematoma requiring transfusion, a case of implant loosening, and 5 categorized as "other." The occurrence of serious device-related adverse events was $2.0 \%(5 / 250)$, within the expected range for this commercially available device. ${ }^{18}$ One event was related to back pain, another to device malposition, both noted above as a procedural event. The other device-related events involved pain in extremity, bilateral SI joint pain, and device loosening. Two of these events resulted in a revision surgery.

\section{DISCUSSION}

Interim results from this prospective, multicenter trial (EVSI) demonstrated that MI SI joint fusion surgery with decortication, bone grafting, and fixation with threaded implants resulted in a statistically significant mean VAS pain score reduction of $56.8 \%$ at 12 months following surgery. The VAS MCID was achieved by $72.2 \%$ of patients at 12 months. 
Table 4. Fusion rates for minimally invasive sacroiliac joint using SImmetry device.

\begin{tabular}{|c|c|c|c|c|}
\hline Study & $N$ & Fusion Rate & Follow-Up & Notes \\
\hline Current study & 201 & $68.7 \%$ & $12 \mathrm{mo}$ & Presence of bony fusion based on independent evaluation of CT imaging at $12 \mathrm{mo}$ \\
\hline Cross et $\mathrm{al}^{21}$ & 19 & $78.9 \%$ & $12 \mathrm{mo}$ & $\begin{array}{l}\text { CT imaging; independently assessed and adjudicated for presence of bridging bone. } \\
\text { Threaded implant fixation with decortication and graft placement }\end{array}$ \\
\hline Kube et $\mathrm{al}^{3}$ & 17 & $88.2 \%$ & $12 \mathrm{mo}$ & $\begin{array}{l}\text { Thin-slice }(<2 \mathrm{~mm}) \text { CT imaging assessment of bony bridging across the sacroiliac joint and } \\
\text { absence of lucency. Threaded implant fixation with decortication and graft placement }\end{array}$ \\
\hline Abassi et $\mathrm{al}^{6}$ & 19 & $73.7 \%$ & $12 \mathrm{mo}$ & $\begin{array}{l}\text { CT imaging; independent assessment of solid bridging bone. Threaded implant fixation with } \\
\text { decortication and graft placement }\end{array}$ \\
\hline
\end{tabular}

Abbreviation: CT, computed tomography.

The improvement in ODI scores by $43.9 \%$ at the 12 month follow-up visit was also statistically significant, reflecting MCID achievement by $62.5 \%$ of patients. These results are consistent with the early 6-month results on the first 50 patients enrolled in this same study reported by Araghi et al. ${ }^{8}$

Araghi et al also reported a substantial reduction in opioid use in the first 50-patient cohort of the EVSI study; prior to surgery, 33 patients $(66 \%)$ were taking opioids, but this number had decreased to 15 patients $(30 \%) 6$ months following surgery. ${ }^{8}$ In the current interim cohort, $62.7 \%$ of patients relied on opioids for pain relief prior to their MI SI joint surgery, decreasing to $26.9 \%$ at 12 months after surgery. These results are significant and appear greater than those seen in clinical studies of other MI SI joint fusion devices, where $58 \%$ of patients were still relying on opioids for pain relief at 6 months and $33 \%$ to $55 \%$ of patients were still using opioids at 24 months following surgery. ${ }^{19,20}$

The patients in this cohort of the EVSI trial achieved fusion in $68.7 \%$ of patients at 12 months postprocedure. The fusion results are comparable to other studies (Table 4).

Joint preparation using the decortication instrument is an integral step in this MI SIJF procedure to provide the environment necessary for facilitating the growth of bridging. The decortication step also produces a cavity sufficient for placement of graft material to aid in the bone formation process. The mean volume of bone graft material placed in this EVSI 12-month cohort was $5.4 \mathrm{cc}$ and ranged between 0 and $20 \mathrm{cc}$. The joint preparation and placement of graft material prior to implantation of the 2 screw-based devices appear to support the generation of bridging bone and SI joint fusion. The results of this study cohort compare favorably with radiographic evidence of fusion rates reported for other MI implants that are used to fixate SI joints but are placed without joint preparation and use of graft material. Duhon et $\mathrm{al}^{20}$ reported a rate of bridging bone of up to $25 \%$ at 12 months following surgery for 159 patients using triangular titanium implants. A more recent study by Dengler et $\mathrm{al}^{22}$ reported that for the 43 patients (66 SI joints) with CT scans available for analysis 12 months after implantation with triangular titanium implants, intraarticular fusion with bridging bone was not commonly observed.

The limitations of this study include the sample size and the limited follow-up. With approximately $80 \%$ follow-up, there is likely to be some degree of induced bias in the results, primarily caused by missed visits, which represent $11.2 \%$ of patients. This report is a 12-month interim analysis of the full study, which will ultimately include evaluation of all outcomes through 24 months postsurgery-every effort will be taken to collect data on some of those who missed 12-month visits for the final 24month analysis to reduce bias as much as possible. These interim results are consistent with the trends observed in an initial analysis of the first 50 patients at 6 months follow-up ${ }^{8}$ and support the continuation of the EVoluSIon study through 2 years of follow-up. Another limitation of this trial is the lack of a control group. Comparisons of other MI SI joint fusion systems to conservative pain management ${ }^{19,23,24}$ and open surgical approaches have already been performed, ${ }^{1,25}$ however, confirming the benefits of the MI approach and minimizing the necessity of including such control groups in additional studies. The purpose of this study is to evaluate outcomes using the system of decortication, grafting, and placement of screwbased implants. While the discussion of these outcomes included a comparison to studies of another MI SI joint fusion system, differences in the study protocols, analysis techniques, and procedures used at investigational sites may all contribute to differences seen in study results.

As with any surgical treatment for SI joint dysfunction, the ultimate goal is long-term pain relief. This interim analysis indicates that the use of decortication, grafting and screw-based implants may achieve that goal for a substantial majority of treated patients.

\section{CONCLUSION}

MI SIJF surgery with decortication, bone graft, and threaded implant fixation results in demonstrated improvement in pain and disability scores through 1 year with few complications. This interim analysis illustrates 
that the procedure can be performed safely, resulting in an average reduction in pain of $56.8 \%$, significant reductions in disability, and also a substantial reduction in the use of opioids. Furthermore, assessment of fusion showed that $68.7 \%$ of patients had bony bridging.

\section{ACKNOWLEDGMENTS}

The authors acknowledge the participating EVoluSIon investigators and study coordinators.

\section{REFERENCES}

1. Smith AG, Capobianco R, Cher D, et al. Open versus minimally invasive sacroiliac joint fusion: a multi-center comparison of perioperative measures and clinical outcomes. Ann Surg Innov Res. 2013;7(1):1-12. doi:10.1186/1750-1164-7-14

2. Polly DW, Cher DJ, Wine KD, et al. Randomized controlled trial of minimally invasive sacroiliac joint fusion using triangular titanium implants vs nonsurgical management for sacroiliac joint dysfunction: 12-month outcomes. Neurosurgery. 2015;77(5):674-690. doi:10.1227/NEU.0000000000000988

3. Kube RA, Muir JM. Sacroiliac joint fusion: one year clinical and radiographic results following minimally invasive sacroiliac joint fusion surgery. Open Orthop J. 2016;10(1):679-689. doi:10.2174/1874325001610010679

4. Cross WW, Delbridge A, Hales D, Fielding LC. Minimally invasive sacroiliac joint fusion: 2-year radiographic and clinical outcomes with a principles-based SIJ fusion system. Open Orthop J. 2018;12(1):7-16. doi:10.2174/1874325001812010007

5. Heiney J, Capobianco R, Cher D. A systematic review of minimally invasive sacroiliac joint fusion utilizing a lateral transarticular technique. Int J Spine Surg. 2015;9:40. doi:10.14444/2040

6. Abbasi H, Hipp JA. The assessment of fusion following sacroiliac joint fusion surgery. Cureus. 2017;9(10):e1787. doi:10.7759/ cureus. 1787

7. MenMuir B, Fielding LC. Revision of minimally invasive sacroiliac joint fixation: technical considerations and case studies using decortication and threaded implant fixation. Int J Spine Surg. 2017;11(1):8. doi:10.14444/4008

8. Araghi A, Woodruff $\mathrm{R}$, Colle $\mathrm{K}$, et al. Pain and opioid use outcomes following minimally invasive sacroiliac joint fusion with decortication and bone grafting: the evolusion clinical trial. Open Orthop J. 2017;11(1):1440-1448. doi:10.2174/1874325001711011440

9. Szadek KM, van der Wurff P, van Tulder MW, Zuurmond WW, Perez RSGM. Diagnostic validity of criteria for sacroiliac joint pain: a systematic review. J Pain. 2009;10(4):354-368. doi:10.1016/j.jpain.2008.09.014

10. Hansen HC, McKenzie-Brown AM, Cohen SP, Swicegood JR, Colson JD, Manchikanti L. Sacroiliac joint interventions: a systematic review. Pain Physician. 2007;10(1):165-184. doi:10.36076/ ppj.2007/10/165

11. Manchikanti L, Abdi S, Atluri S, et al. An update of comprehensive evidence-based guidelines for interventional techniques in chronic spinal pain. Part II: guidance and recommendations. Pain Physician. 2013;16(2 Suppl):S49-283. doi:10.36076/ppj.2013/16/ S49
12. Beaubien B, Salib RM, Fielding LC, Block JE. SImmetry sacroiliac joint fusion system with sImmetry decorticator. Surg Sci. 2015;06(7):282-291

13. Miller LE, Block JE. Minimally invasive arthrodesis for chronic sacroiliac joint dysfunction using the sImmetry SI joint fusion system. Med Devices. 2014;7(1):125-130. doi:10.2147/ MDER.S63575

14. Shih YC, Beaubien BP, Chen Q, Sembrano JN. Biomechanical evaluation of sacroiliac joint fixation with decortication. Spine J. 2018;18(7):1241-1249. doi:10.1016/j.spinee.2018.02.016

15. EuroQol Group. EuroQol--a new facility for the measurement of health-related quality of life. Health Policy. 1990;16(3):199-208. doi:10.1016/0168-8510(90)90421-9

16. Brooks R, De Charro F. EuroQol: the current state of play. Health Policy. 1996;37(1):53-72. doi:10.1016/01688510(96)00822-6

17. Salkind N. McNemar test for significance of changes. Encyclopedia of Measurement and Statistics. Thousand Oaks; 2007:2. 576-580. doi:10.4135/9781412952644. http://methods. sagepub.com/reference/encyclopedia-of-measurement-and-statistics

18. Shamrock AG, Patel A, Alam M, Shamrock KH, Al Maaieh M. The safety profile of percutaneous minimally invasive sacroiliac joint fusion. Global Spine J. 2019;9(8):874-880. doi: $10.1177 / 2192568218816981$

19. Polly DW, Swofford J, Whang PG, et al. Two-year outcomes from a randomized controlled trial of minimally invasive sacroiliac joint fusion vs. non-surgical management for sacroiliac joint dysfunction. Int J Spine Surg. 2016;10(2016):28. doi:10.14444/3028

20. Duhon BS, Bitan F, Lockstadt H, et al. Triangular titanium implants for minimally invasive sacroiliac joint fusion: 2-year follow-up from a prospective multicenter trial. Int J Spine Surg. 2016;10:13. doi:10.14444/3013

21. Cross WW, Delbridge AE, Hales DD. Evaluation of Fusion after Minimally Invasive Sacroiliac Joint Fusion Surgery with Decortication: 24-Month Results. 2017.

22. Dengler J, Duhon B, Whang P, et al. Predictors of outcome in conservative and minimally invasive surgical management of pain originating from the sacroiliac joint: a pooled analysis. Spine. 2017;42(21):1664-1673. doi:10.1097/BRS.0000000000002169

23. Dengler JD, Kools D, Pflugmacher R, et al. 1-year results of a randomized controlled trial of conservative management vs. minimally invasive surgical treatment for sacroiliac joint pain. Pain Physician. 2017;20(6):537-550. doi:10.36076/ppj.20.5.537

24. Vanaclocha VV. Minimally invasive sacroiliac joint arthrodesis: experience in a prospective series with 24 patients. J Spine. 2014;03(5):1-7. doi:10.4172/2165-7939.1000185

25. Ledonio CGT, Polly DW, Swiontkowski MF, Cummings JT. Comparative effectiveness of open versus minimally invasive sacroiliac joint fusion. Med Devices. 2014;7(1):187-193. doi:10.2147/ MDER.S60370

Funding: This work was sponsored with institutional support by Surgalign Spine Technologies (Deerfield, IL).

Declaration of Conflicting Interests: Drs Kucharzyk, Colle, Boone, and Araghi are paid consultants for Surgalign Spine Technologies. 
Patient Consent for Publication: Written informed consent was obtained from all patients prior to enrollment.

Ethics Approval: This study was approved by the institutional review board of each participating hospital or if one did not exist, by a central institutional review board, Western IRB.

Disclosure: Drs Kucharzyk, Colle, Boone, and Araghi are investigators in the EVSI study, which is sponsored by Surgalign.

Data Availability Statement: All procedures performed involving human participants were in accordance with the Helsinki Declaration of 1975, as revised in 2008. (https://www.wma.net/en/20activities/ 10ethics/10helsinki/)

Corresponding Author: Christopher Boone, Proliance Orthopaedics \& Sports Medicine, 1231 116th Ave NE, Bellevue, WA, USA; boone34@gmail.com

Published 21 February 2022

This manuscript is generously published free of charge by ISASS, the International Society for the Advancement of Spine Surgery. Copyright () 2022 ISASS. To see more or order reprints or permissions, see http:// ijssurgery.com. 